\title{
Evaluation of a Protocol for fMRI Assessment Associated with Augmented Reality Rehabilitation of Stroke Subjects
}

\author{
Gilda Aparecida de Assis \\ Department of Computing and Systems \\ Federal University of Ouro Preto \\ João Monlevade, Brazil \\ gildaaa1@gmail.com
}

\author{
Alexandre Fonseca Brandão \\ Institute of Physics Gleb Wataghin \\ Brazilian Institute of Neuroscience and Neurotechnology \\ University of Campinas \\ Campinas, Brazil \\ brandaobiotec@gmail.com
}

\author{
Ana Grasielle Dionisio Corrêa \\ Postgraduate in Developmental Disorders \\ Faculty of Computer and Information Technology \\ Mackenzie Presbyterian University \\ São Paulo, Brazil \\ ana.correa@mackenzie.br
}

\author{
Gabriela Castellano \\ Institute of Physics Gleb Wataghin \\ Brazilian Institute of Neuroscience and Neurotechnology \\ University of Campinas \\ Campinas, Brazil \\ gabriela@ifi.unicamp.br
}

\begin{abstract}
New technologies for rehabilitation involving Augmented Reality (AR) as a complement to conventional therapy have appeared in recent years. An earlier study for shoulder rehabilitation using the AR NeuroR computer system showed improved clinical outcomes for stroke patients. This study aims to analyze a proposed protocol to measure possible changes in functional brain connectivity associated with the use of the NeuroR system in the context of shoulder motor rehabilitation of post-stroke subjects. A pilot study was conducted with a poststroke patient, using resting-state functional magnetic resonance imaging (RS-fMRI). RS-fMRI signals were acquired pre and post use of the NeuroR system (pre-test and post-test), integrated into the patient's rehabilitation program. Functional connectivity analysis of RS-fMRI was performed using the motor area as seed. he maximum connectivity value in the pre-test occurred in the ipsilesional parietal region while the maximum in the post-test was located in the ipsilesional frontal region. It was observed that the regions strongly associated with motor activity had higher connectivity values at post-test compared to pre-test. The proposed protocol is suitable and safe for verifying if functional brain connectivity was changed after the rehabilitation program with NeuroR training, indicating a possible neuroplasticity effect. Tests with a larger number of patients are still necessary.

Index Terms-Functional brain connectivity, augmented reality, motor rehabilitation
\end{abstract}

\section{INTRODUCTION}

Neurological injury after stroke frequently leads to hemiparesis or partial paralysis and, despite conventional rehabilitation therapies, most stroke survivors report disability in at least one activity of daily living. Stroke is one of the leading causes of permanent disability with approximately 15 million strokes each year worldwide [1]. Post-stroke rehabilitation has focused on reducing motor impairment and minimizing physical disability since the motor impairments that follow a stroke can be reduced depending on the number of sessions, type, and intensity of rehabilitation [2].

In recent years, new technologies for rehabilitation involving virtual environments have received attention from the scientific community. These technologies are being investigated to develop more effective strategies to help recover from physical disabilities resulting from stroke damage [3][5]. Indeed, the effect of treatment using Virtual Reality (VR) applications in the recovery of cognitive function, gait, balance, and activities of daily living (ADLs) in patients who have suffered a stroke has been evaluated and there is statistically significant evidence of improvement regarding the use of VR for arm function and ADLs [6]. VR offers a digital version of a real-life setting, while Augmented Reality (AR) delivers virtual elements as an overlay to the real world. AR has emerged as a potential method in the field of rehabilitation, with the advantages of allowing a controllable environment and salient feedback, inducing the sense of presence, producing entertaining treatments and providing digital records of the rehabilitation session. This last feature allows for an individualized model of motor and cognitive learning, appropriate for each patient [7].

An AR system was proposed in [8] based on the assumption that a controlled amplification of the movement of an impaired limb can lead to improvement of motor movement simply playing a virtual memory game. It has been reported that therapy using VR games should improve patient motivation through graded task success [5]. Previous works in the use of games to increase motivation in chronic stroke patients training their upper extremity were presented in [9]-[11].

Rehabilitation therapy requires repetitions and long training 
periods, which may also produce boredom, reducing the effectiveness of the therapy by diminishing patient participation and engagement [5]. VR training systems have the potential to quantify therapy procedures and improve patient motivation using real-time task evaluation and reward [4]. A study using an interactive workbench called VR-Mirror and combining physical and mental practice for post-stroke rehabilitation was presented in [12], [13]. Measurements of wrist function revealed increases in the range of motion and the grip strength of the affected limb of the post-stroke patient [13].

The promising results of VR-Mirror combining physical and mental practice with VR technology applied to motor rehabilitation supported the proposal of the NeuroR system. The aim of this work was to investigate if the proposed protocol including functional Magnetic Resonance Imaging (fMRI) and NeuroR training is appropriate to highlight the brain dynamics taking place before and after application of a NeuroR training protocol. This understanding could provide support for the adoption of NeuroR as another resource in rehabilitation scenarios. Hence, and more specifically, this study was proposed in order to assess the feasibility of the proposed protocol by measuring the functional brain connectivity of a small number of brain regions of interest (ROIs), evaluated from resting-state functional Magnetic Resonance Imaging (RS-fMRI) data of a post-stroke patient using NeuroR training incorporated into his rehabilitation program.

\section{PRoposed PRotocol}

\section{A. NeuroR System}

NeuroR was proposed for upper-limb post-stroke rehabilitation using AR combined with mental practice. In the NeuroR system, training works in the following way. The physiotherapist guides the patients so that they do the impaired upper-limb task in the mirrored AR environment, receiving visual feedback from the virtual arm on the screen. The virtual arm is a tridimensional (3D) arm generated by computer, superimposed on the captured image from a webcam in real time, which executes shoulder animations triggered by either the physiotherapist or bioelectrical (electromyography) signals of the patient (Fig. 1). NeuroR aims to provide motor imagery for the stroke patient using the virtual 3D arm that replaces the paralyzed arm in an actual image of the patient on the screen [14]. Studies have indicated that motor imagery belongs to the same category of brain processes involved in actually programming actions without any motor output [15]. Clinical studies with NeuroR showed that participants presented evidence of improvement in shoulder movements after training with the system, indicating promising results for upper-limb motor rehabilitation of patients that had suffered a stroke more than a year before [16].

\section{FMRI DATA}

Typically, an fMRI study is performed while the patient is performing a motor or cognitive task, or while he/she is subjected to a sensorial stimulus (task fMRI). However, motor deficits can severely impair task performance of the patient and
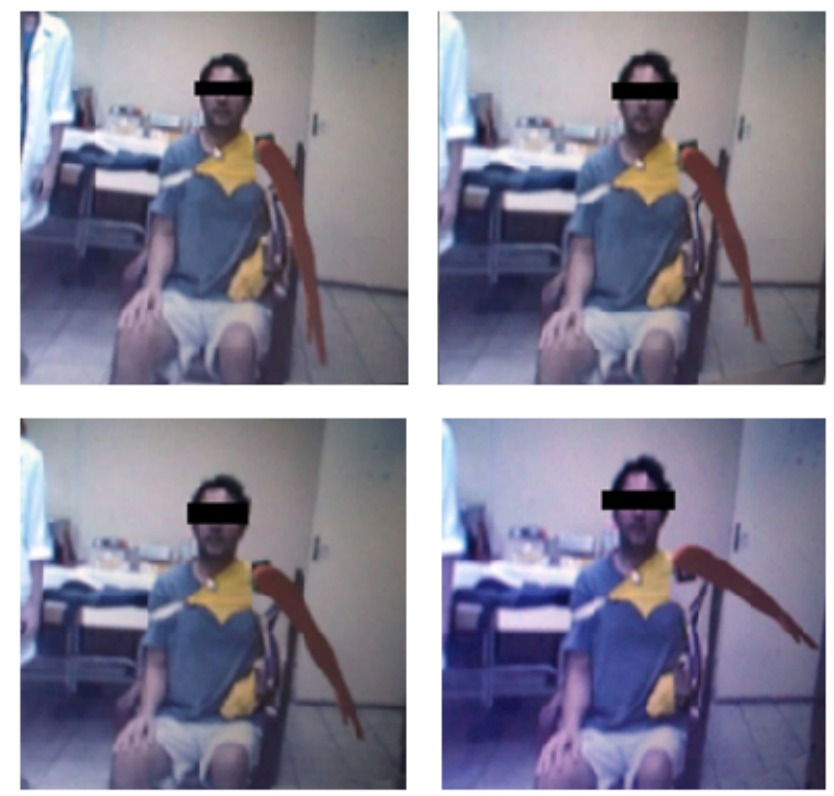

Fig. 1. Sequence of video frames showing exercise performed by NeuroR.

introduce false positive or false negative brain activations. As a workaround, studies have been carried out with patients in resting state (without the subject performing a specific task in the scanner). At rest, brain regions exhibit spontaneous fluctuations at low frequencies in the blood oxygenation leveldependent (BOLD) signal [17], [18].

Resting-state fluctuations in the BOLD signal (resting state fMRI, or RS-fMRI) may present valuable data for delineating the human neural functional architecture. Consistently, large-scale spatial patterns of human brain signals have been identified using both fMRI and positron emission tomography [19]. A large number of studies have been focused on the connectivity patterns between multiple ROIs within spatially distributed, large-scale networks, characterized via both model-driven (seed-based correlation analysis SCA) and data-driven analyses (independent component analysis ICA). These connectivity patterns have been designated as intrinsic connectivity networks or resting-state networks (RSNs). They reflect neuron populations firing together with a common functional purpose. RSNs can be detected at individual subject and group levels across several analysis techniques [19].

Many RSN maps have been identified using ICA [20], including the sensory and motor network, which are important for this work. The occurrence of these networks depends on the nature of neural processes evoked or induced, being consistent with the stimulus-evoked co-activation patterns. In our investigation, we used the SCA technique to reveal functional connectivity in the post-stroke patient's brain, before and after the motor rehabilitation program supplemented by the NeuroR training. The SCA method requires a priori selection of a voxel, cluster or brain region (ROI) based on previous literature, to serve as a seed. Time series data are extracted from the seed and all remaining brain voxels, and the Pearson 
correlation between these voxels and the seed is calculated. SCA approaches show the network of brain regions that are functionally and strongly connected to the seed [21].

In [22], fifteen healthy right-handed subjects underwent transcranial magnetic stimulation (TMS) and fMRI scanning where they performed right wrist flexion and right ankle dorsiflexion in the scanner. Results showed that the ipsilateral primary motor cortex plays a central role in unilateral motor executions. This study revealed that the ipsilateral and contralateral primary motor cortex belonged in the same motor network during a simple, unilateral muscle contraction in the upper limb. Given these results, in our work, we investigate two questions. The first is whether NeuroR training is suitable for use in a rehabilitation program. The second is whether the fMRI assessment is able to detect alterations in the functional brain connectivity between the uninjured motor cortex and the whole brain, comparing pre and post-test data.

\section{Methods}

\section{A. Subject}

A right-handed male patient aged 47 years, with a clinical diagnosis of stroke 10 months beforehand, participated in the study. He had motor impairment of the upper limb (right hemiplegia) and aphasia. The patient signed an informed consent, approved by the Ethics Committee of the University of Campinas, Brazil.

\section{B. Augmented Reality System}

The NeuroR computer software was designed to support shoulder motor rehabilitation of patients who suffered a stroke. The NeuroR software provides visual feedback for physical and mental practice using the AR environment. NeuroR blends live images with virtual objects. Patients see their own image in real time on a projection screen with their injured arm replaced by a 3D virtual arm. For each frame, an alpha map is used to classify all pixels into background or foreground (glove). The virtual arm performs computer animations compatible with shoulder flexion and shoulder abduction. A simple approach to image composition during the virtual arm animation using two background frames, one without and another with the user was proposed [23]. Two event triggers were provided to start the virtual arm animation. These were keyboard keys and data collected with an electromyography device where surface applied electrodes were attached to the muscles of the injured limb. NeuroRs setup includes a tripod, a webcam, a multimedia projector (Fig. 2a), a glove dressing the injured arm (Fig. 2b) and a fiducial marker attached to the injured shoulder of the patient (Fig. 2c).

A physiotherapist instructs the patients to perform the required movements using the injured arm, while they are facing the projection screen displaying their image. The image of the arm glove on the projection screen is replaced by a three-dimensional arm. Such replacement is done using image processing techniques to detect the gloves region from an image frame [14], [16]. From the point when the rehabilitation session starts, the participants do not see the injured arm

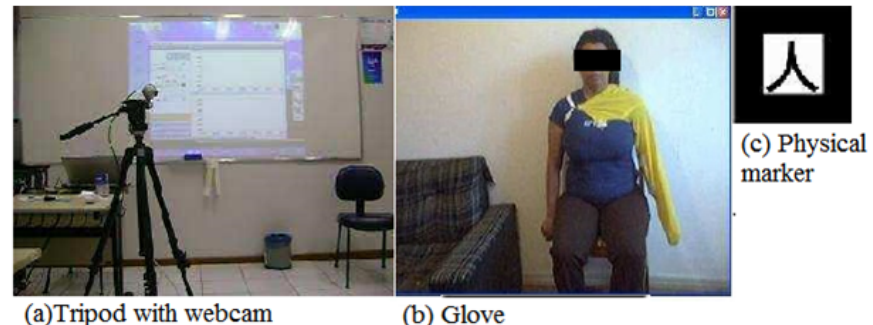

Fig. 2. NeuroRs setup.

wearing the glove on the screen. Instead, they see a virtual 3D arm replacing the actually injured limb on the screen. During the rehabilitation session, the patients can stop and rest, if necessary.

\section{Experimental Procedure}

Before the beginning of the rehabilitation sessions, the patient underwent an RS-fMRI acquisition. The patient performed sessions of conventional therapy (treatment that is widely accepted and used by most healthcare professionals) supplemented by the NeuroR system training for two weeks, twice a week, totaling four training sessions. For the NeuroR training, the patient performed 10 repetitions of shoulder abduction and also 10 repetitions of shoulder flexion, both exercises without assistance, in a sitting position. The physiotherapist verbally instructed the patient regarding the exercise to be performed. In the NeuroR training sessions, the keyboard triggered the animation of the virtual arm, and the first author of this paper played the external observers role. All the observations were transcribed right after the end of the sessions. For the upper-limb conventional rehabilitation therapy, the following exercises were adopted: shoulder elevation without assistance, mobilization of shoulder and shoulder abduction, mobilization of shoulder, shoulder flexion and shoulder extension, internal rotation and external rotation of the shoulder with assistance, and weight bearing on the upper limb. Each of the exercises was repeated 10 times per session.

\section{RS-fMRI Data Processing}

Off-line fMRI data processing was performed using SPM $12^{1}$ and the UF2C toolbox ${ }^{2}$, run within the MATLAB platform. We pre-processed the image data and performed the statistical analysis according to the UF2C standard pipeline [24]. Pre-processing was based on: definition of the anterior commissure as the origin of the reference system of the structural and RS-fMRI images, fMRI volumes realignment (using the mean image as reference), images registration (fMRI mean image with T1-WI), spatial normalization of all images to a standard space, spatial smoothing of fMRI images, T1-WI tissue segmentation (gray matter, white matter, and cerebral spinal fluid). Additionally, we regressed out six head motion

\footnotetext{
${ }^{1}$ http://www.fil.ion.ucl.ac.uk/spm

${ }^{2}$ http://www.lni.hc.unicamp.br/app/uf2c/
} 
parameters (three rotational and three translational) as well as the white matter and cerebral spinal fluid average signals. Finally, we band-pass filtered $(0.0080 .1 \mathrm{~Hz})$ the time-series. We chose as seed for functional connectivity a region of interest (ROI) of 444 voxels located in the right pre-central area (non-injured area) according to the AAL atlas [25] (Fig. 3). The ROI was located in the primary motor cortex (Brodmann area 4) in the precentral gyrus, which is a prominent structure on the surface of the posterior frontal lobe. We selected this ROI in order to evaluate functional connectivity in brain areas related to motor networks. We computed the average time series of all ROI voxels within the subjects gray matter (as found in the tissue segmentation step) to use as seed. Pearson's correlation scores were calculated between the seeds time series and the time series of all gray matter voxels of the brain. In order to evaluate and characterize the differences between pre-test and post-test data, the average correlation values of each AAL Atlas region (116 regions) were computed. This was done by converting these values to $\mathrm{Z}$-scores (Fishers $\mathrm{Z}$ transformation), calculating the mean value, and transforming them back to the correlation space.

\section{RESULTS}

We assessed the internetworks connectivity looking at positive and negative correlations between the RS-fMRI data from whole brain and healthy motor seed at pre- and post-test acquisitions, shown in Fig. 3.

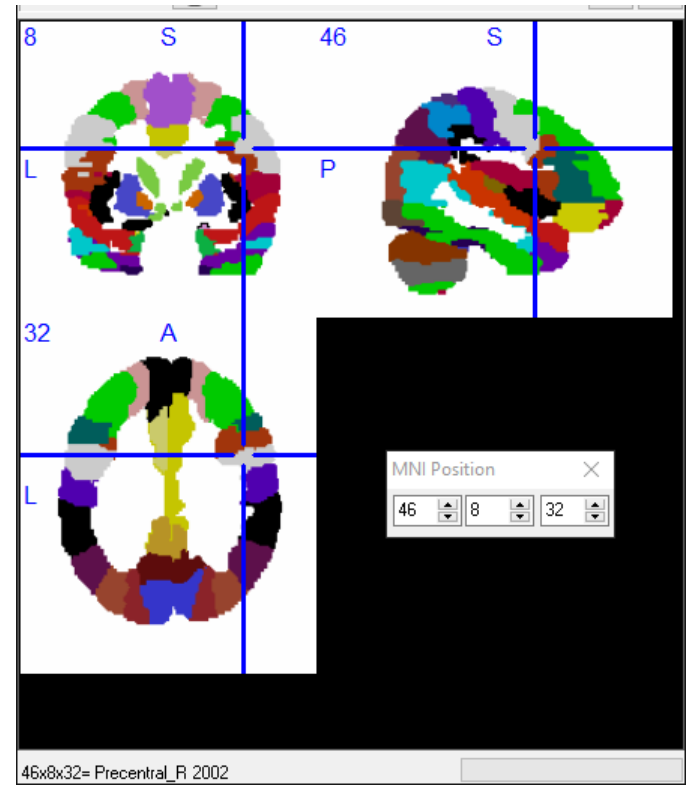

Fig. 3. ROI (in grey) corresponding to the right pre-central area in the AAL Atlas, used as a seed for functional connectivity estimation.

Fig. 4 shows the structural 3D T1-weighted data acquired in the pre- and post-test. There were no visible changes in the lesion region in the left hemisphere of the brain between the pre-test and post-test acquisitions.

The mean positive correlation between all brain voxels and the seed was $0.20 \quad 0.18$ in the pre-test and $0.20 \quad 0.15$ in

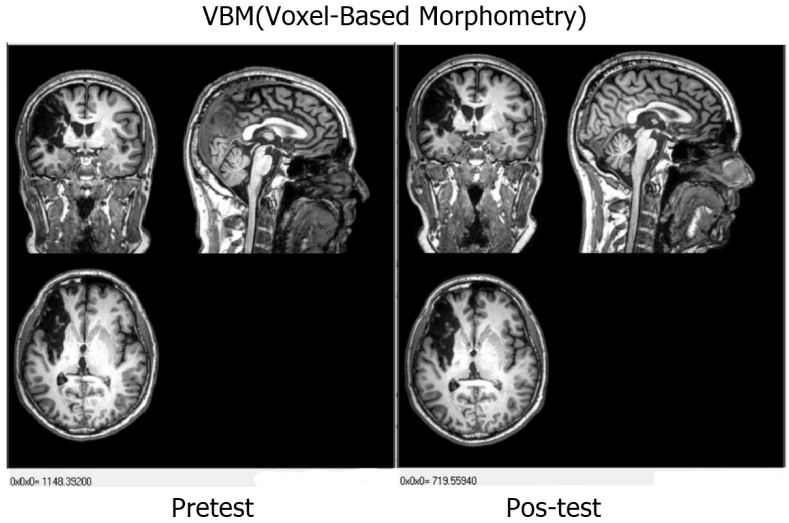

Fig. 4. Anatomical (or structural) brain images acquired in the pre-test (left) and post-test (right).

the post-test. The mean negative correlation between all brain voxels and the seed was $-0.16 \quad 0.12$ in the pre-test and -0.19 0.12 in the post-test. There were no differences between the pre-test and post-test for the positive correlations (T-Test, twotailed, $\mathrm{p}$-value $=0.28462$, alpha $=0.05)$. However, significant differences were found between the pre-test and post-test for the mean negative correlations $(\mathrm{T}$-Test, two-tailed, $\mathrm{p}$-value $=$ 0.0001 , alpha $=0.05$ ). This result means that the post-test either presented a larger number of voxels inversely correlated with the seed (healthy motor area) or increased amplitude of the negative correlation values of these voxels.

For the pre-test, the highest positive Pearsons correlation scores were achieved in the inferior parietal lobe in the left hemisphere (Parietal-Inf-L of AAL Region 61), whereas for the post-test, the highest scores were for the left frontal operculum in the inferior frontal lobe (Frontal-Inf-Oper-L of AAL Region 11 in Fig. 5).

\section{DISCUSSION}

Several techniques have been used in the motor recovery process of the upper and lower limbs of stroke patients, where intensive and repeated training of specific task gives satisfactory results [26]. But it is still a challenge to implement such techniques in a real environment, such as in rehabilitation clinics and hospitals, due to the loss of interest by patients in repetitive tasks. For this reason, rehabilitation programs based on VR/AR have been highlighted as an alternative therapy for motor recovery [26]. However, there are still very few publications addressing the brain effects caused by this type of rehabilitation programs. Some previous works encompassing AR or VR and RS-fMRI have been summarized in Table I. From these, apart from the work of our group [27], no other study investigated the effect of VR/AR motor rehabilitation techniques in the brain using RS-fMRI. All other publications addressed different issues, such as social behavior [28], internet gaming disorder [29], spatial navigation [30] and spatial learning [31]. Only the paper by [32] proposed a study more similar to the one presented here, addressing 
rehabilitation of children with post-neonatal acquired brain injury but, the results have not been published yet.

In our work, we proposed the supplementary AR motor rehabilitation system NeuroR, and assessed brain changes following the rehabilitation procedure using RS-fMRI. The mean positive and negative correlation between all brain voxels and the seed (illustrated in Fig. 4) shows that the post-test either presented a larger number of voxels inversely correlated with the seed (healthy motor area) or increased amplitude of the negative correlation values of these voxels. Although we cannot specify what might have occurred, these results present functional connectivity alterations between the preand post-test. Particularly, functional connectivity increased in interhemispheric motor areas (Fig. 6), which agrees with the work of Carter et al., showing that disrupted interhemispheric functional interactions might underlie behavioral motor deficits and determine post-stroke recovery [33].

Cerebral connectivity between brain regions can be measured in two ways: during a specific task and at resting state. In this study, a subject underwent resting-state fMRI scanning. During resting state, the subject is instructed to remain immobile (motionless) in the scanner without thinking about anything specific [34]. As mentioned, mean correlation values for every region of the AAL Atlas (with the seed) were obtained, in order to better analyze possible differences between the pre- and post-test. Fig. 5 shows a plot of the correlation values for these regions, for pre-test (blue) and post-test (red).

As observed in Fig. 5, the highest positive Pearsons correlation scores were achieved in the inferior parietal lobe in the left hemisphere (region 61 in Fig. 5) for the pre-test, whereas for the post-test, the highest scores were for the left frontal operculum in the inferior frontal lobe (region 11 in Fig. 5). The inferior parietal lobe belongs to the default mode network (DMN), which is anti-correlated with cognitively demanding tasks, such as a visuospatial working memory task [35] [36]. The DMN generally shows strong functional connectivity during resting state scans. On the other hand, the frontal operculum in the inferior frontal lobe is part of the task control system; it is described as the core of the stable maintenance of task mode and strategy system, and it is thought to instantiate, maintain and adjust a task during its performance [37]. These results suggest a shift in functional connectivity from regions that are most active when no external tasks demand attention to regions more related to movement planning. This is consistent with neuroplasticity occurring in the patients brain, in order to regain motor control.

In addition, the second result shows that most regions of the cerebellum (12 out of 18) presented higher functional connectivity in the post-test than in the pre-test (Fig. 7). Studies in humans have implicated the sensorimotor cortex, premotor cortex, supplementary motor area, superior parietal cortex, striatum, and cerebellum in motor execution, planning, and learning [35] [36] [38]. Therefore, this result also points to possible neuroplastic alterations occurring in the patients brain, as he regains his motor skills.

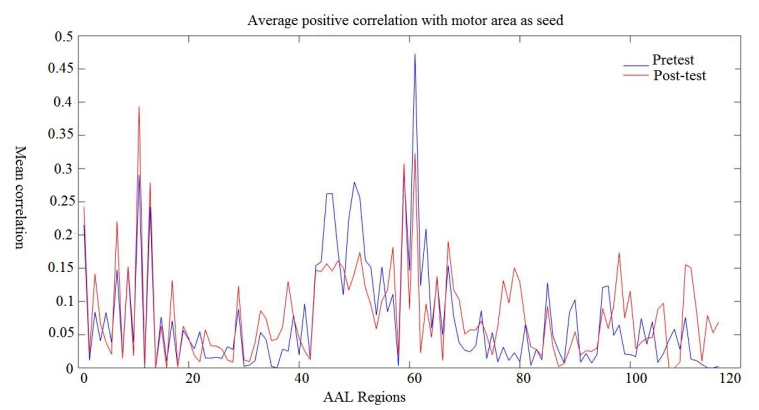

Fig. 5. Mean correlation values between AAL regions and seed (primary motor cortex).

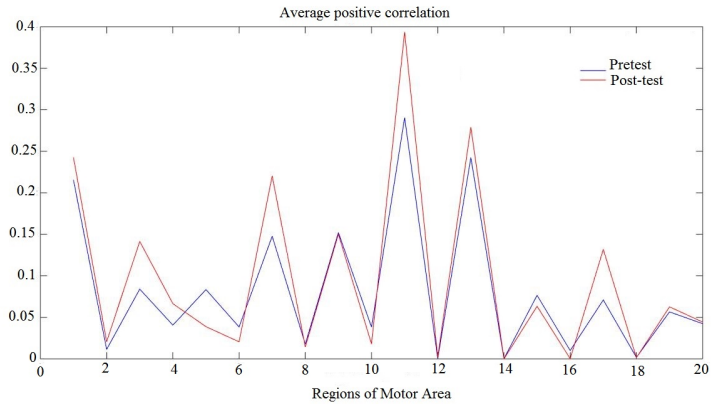

Fig. 6. Zooming a motor area of Fig. 5.

Overall, the results achieved can be due to training with NeuroR, or due only to the conventional rehabilitation, or to a combination of both. Despite the positive results of this study, further work will apply this methodology across patients receiving NeuroR training using appropriate control groups to achieve more accurate outcomes. Other points to be addressed in prospective studies are the short period of time used for training with NeuroR and the small number of repetitions with NeuroR compared to other rehabilitation therapies. For instance, in robotic therapy for upper limb rehabilitation with InMotion $^{3}$, the patients perform about 1,200 repetitions per session, while in the NeuroR training 20 repetitions were carried out per session. However, it is important to stress

\footnotetext{
${ }^{3} \mathrm{http} / / /$ bionikusa.com/healthcarereform/upper-extremity-rehabilitiation/ inmotion2-arm/
}

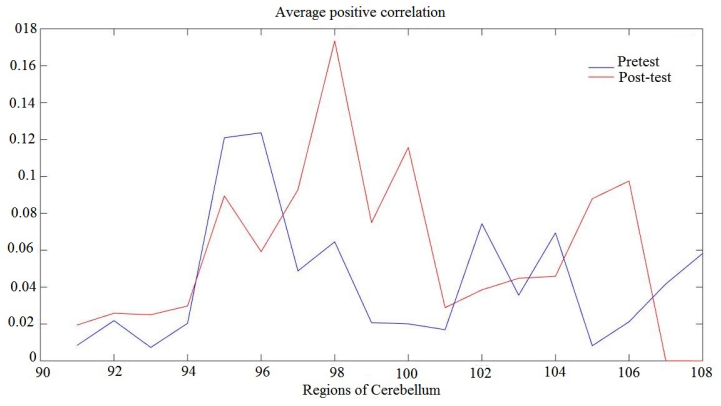

Fig. 7. Zooming a cerebellum area of Fig. 5. 
TABLE I

VR/AR EXPERIMENTS WITH RS-FMRI DATA

\begin{tabular}{|c|c|c|c|c|c|}
\hline $\begin{array}{l}\text { Author } \\
\text { (Year) }\end{array}$ & $\begin{array}{l}\text { AR/VR } \\
\text { study }\end{array}$ & Sample size & Type of analysis & Main purpose & Finding/Additional information \\
\hline [28] & VR & 43 healthy adults & $\begin{array}{l}\text { fMRI data were decomposed } \\
\text { into components using Inde- } \\
\text { pendent Component Analysis } \\
\text { (ICA). ICA of the selfish and } \\
\text { altruistic groups were com- } \\
\text { pared according to some rest- } \\
\text { ing state networks. }\end{array}$ & $\begin{array}{l}\text { To investigate a complex social } \\
\text { behavior analyzing functional } \\
\text { connectivity within brain net- } \\
\text { works of participants who ex- } \\
\text { hibited either a selfish behavior } \\
\text { or an altruistic one in a virtual } \\
\text { building on fire. }\end{array}$ & $\begin{array}{l}\text { The VR scenario was experi- } \\
\text { enced from a first-person per- } \\
\text { spective using fMRI-compatible } \\
\text { goggles and earphones. }\end{array}$ \\
\hline$[29]$ & VR & $\begin{array}{l}24 \text { adults diagnosed } \\
\text { with internet gaming } \\
\text { disorder }\end{array}$ & $\begin{array}{l}\text { Functional connectivity from } \\
\text { posterior cingulate seed to } \\
\text { other brain areas was investi- } \\
\text { gated using RS-fMRI. }\end{array}$ & $\begin{array}{l}\text { To compare cognitive behavior } \\
\text { therapy (baseline group) against } \\
\text { VR therapy (test group) for sub- } \\
\text { jects with internet gaming dis- } \\
\text { order. }\end{array}$ & $\begin{array}{l}\text { Both groups showed reduced } \\
\text { connectivity in cortico-striatal- } \\
\text { limbic circuit. }\end{array}$ \\
\hline$[30]$ & AR & 44 healthy adults & $\begin{array}{l}\text { RS-fMRI volumes were ana- } \\
\text { lyzed using a seed-driven ap- } \\
\text { proach. The connectivity of } \\
\text { the right hipocampal region } \\
\text { to the remaining atlas regions } \\
\text { (ROI to ROI) was estimated } \\
\text { by computing Pearson corre- } \\
\text { lation coefficients. }\end{array}$ & $\begin{array}{l}\text { To investigate possible changes } \\
\text { in functional connectivity of } \\
\text { brain regions involved in spa- } \\
\text { tial navigation comparing par- } \\
\text { ticipants who used AR glasses } \\
\text { with GPS navigation against } \\
\text { participants who had not used } \\
\text { any GPS devices for a period. }\end{array}$ & $\begin{array}{l}\text { The outcomes suggest a possi- } \\
\text { ble harmful effect of long-term } \\
\text { usage of GPS technology on our } \\
\text { brain functioning when the nav- } \\
\text { igation process is externalized } \\
\text { to AR glasses. }\end{array}$ \\
\hline [32] & VR & $\begin{array}{l}60 \text { children will be en- } \\
\text { rolled in the study }\end{array}$ & $\begin{array}{l}\text { All participants will perform } \\
\text { motor tasks during fMRI ac- } \\
\text { quisitions. }\end{array}$ & $\begin{array}{l}\text { An additional acquisition of } \\
\text { RS-fMRI data will also be col- } \\
\text { lected for analysis of func- } \\
\text { tional connectivity after the mo- } \\
\text { tor paradigms. }\end{array}$ & $\begin{array}{l}\text { To propose a design for evalua- } \\
\text { tion of a web-based VR therapy } \\
\text { that comprises upper limb and } \\
\text { cognitive training to children } \\
\text { with acquired brain injury in the } \\
\text { post-neonatal period. Results of } \\
\text { the experiment have not been } \\
\text { published yet. }\end{array}$ \\
\hline [31] & VR & 10 healthy volunteers & $\begin{array}{l}\text { RS-fMRI connectivity mea- } \\
\text { sures of the brain areas known } \\
\text { for spatial exploration and vi- } \\
\text { suospatial processing (ROIs) } \\
\text { were correlated to perfor- } \\
\text { mance of the participants. }\end{array}$ & $\begin{array}{l}\text { In each of these ROIs, the } \\
\text { BOLD time courses were aver- } \\
\text { aged and then correlated with } \\
\text { every voxel within the brain. } \\
\text { To investigate the relationship } \\
\text { between the unsupervised spa- } \\
\text { tial learning performance in an } \\
\text { immersive VR environment and } \\
\text { RS-fMRI BOLD signal vari- } \\
\text { ability in multiple brain regions } \\
\text { associated with learning and } \\
\text { memory. }\end{array}$ & $\begin{array}{l}\text { Results showed that the resting- } \\
\text { state functional connectivity be- } \\
\text { tween the left caudate and } \\
\text { brain regions responsible for } \\
\text { visuospatial object processing } \\
\text { and attention is correlated with } \\
\text { learning performance. }\end{array}$ \\
\hline [27] & VR & 13 healthy subjects & $\begin{array}{l}\text { RS-fMRI scans were per- } \\
\text { formed before and after the } \\
\text { VR gestural interaction. }\end{array}$ & $\begin{array}{l}\text { A seed-based analysis was per- } \\
\text { formed to correlate the RS- } \\
\text { fMRI signals of the ROI in the } \\
\text { primary motor cortex and all } \\
\text { other voxels in the brain. }\end{array}$ & $\begin{array}{l}\text { To evaluate the outcome of pa- } \\
\text { tients engaged in VR training in } \\
\text { order to provide motor stimuli } \\
\text { for the upper limbs, There were } \\
\text { no significant changes in mo- } \\
\text { tor networks connectivity be- } \\
\text { fore and after the VR session. }\end{array}$ \\
\hline
\end{tabular}

that this was a pilot study aiming to evaluate the feasibility and potential effectiveness of the proposed protocol using the NeuroR system added to conventional rehabilitation and fMRI to detect alterations in the functional brain connectivity. In this sense, the NeuroR training was found to be simple to be embedded into the rehabilitation program, with promising initial results. In addition, the fMRI assessment was able to detect brain changes in functional connectivity, possibly associated with the undertaken rehabilitation program.

\section{CONCLUSION}

A pilot study is an essential initial step in exploring a novel intervention or a new application of an intervention. The key role of a pilot study is to investigate the feasibility of a research experimental procedure to be used in a larger scale efficacy study. Several aspects can be examined such as recruitment, inclusion and exclusion criteria, duration of the interventions/trainings, treatment adherence and assessment procedures. Well-designed and carried out pilot studies reduce the risk of several problems that are commonly faced in clinical trials [39].

In conclusion, the tests carried out in this pilot study demonstrated that NeuroR training could be integrated into a conventional rehabilitation program with little adjustments. Regarding the use of fMRI as an assessment tool for brain changes resulting from the rehabilitation process, the results point to the feasibility of using this tool, since changes in functional brain connectivity between motor areas were re- 
vealed after performing "conventional rehabilitation" together with NeuroR training. The small number of repetitions in the NeuroR training, the fact that this training was conducted in conjunction with conventional therapy, besides the fact that tests were carried out with only one patient, do not allow to state that the results were exclusively due to the NeuroR training. On the other hand, this was just a feasibility study.

Naturally, further studies with more patients need to be performed. These patients will be subjects who have already undergone the rehabilitation program and have reached the plateau of functional gains and stalled progress, regardless of the time following a stroke. Such a study is expected to be able to evaluate the use of the NeuroR training by itself, for the rehabilitation of patients after stroke. Also, improvements regarding clinical outcomes are not examined in this study. Fugl-Meyer Assessment and Modified Ashworth scale should be included in future studies to assess possible changes in the motor function of the stroke patients and to assess possible relations between these variables and the functional connectivity findings, which may provide biomarkers for rehabilitation success.

\section{ACKNOWLEDGMENT}

We thank CRR - Reference Center for Rehabilitation of Campinas, Campinas, Brazil, for patient recruitment and rehabilitation support, and FAPESP-Brazil (So Paulo Research Foundation), for financial support (grants 2013/07559-3 and 2015/23830-4).

\section{REFERENCES}

[1] M. Steinisch, M.G. Tana, and S. Comani, "A PostStroke Rehabilitation System Integrating Robotics, VR and HighResolution EEG Imaging", IEEE Transactions On Neural Systems And Rehabilitation Engineering, vol. 21, n. 5, pp. 849-859, 2013, Sept 2013.

[2] E. Taub, G. Uswatte and D.M. Morris, "Improved motor recovery after stroke and massive cortical reorganization following Constraint-Induced Movement therapy", Physical Medicine and Rehabilitation Clinics of North America, vol. 14(1 Suppl), pp. 77-91, Feb 2003.

[3] G. C. Burdea, "Virtual Rehabilitation-benefits and Challenges, Methods of Information in Medicinevol, vol. 42, n. 5, pp. 519-523, 2003.

[4] C. Schuster-Amft, A. Henneke, B. Hartog-Keisker, L. Holper, E. Siekierka, E. Chevrier, P. Pyk, S. Kollias, D. Kiper and K. Eng, "Intensive virtual reality-based training for upper limb motor function in chronic stroke: a feasibility study using a single case experimental design and fMRI", Disabil Rehabil Assist Technol, vol. 10, n. 5, pp. 385-392, 2015.

[5] G. C. Burdea, C. Cioi, J. Martin, D. Fensterheim and M. Holenski, "The Rutgers arm ii rehabilitation systema feasibility study", IEEE Trans Neural Syst Rehabil Eng, vol. 18, n. 5, pp. 505-514, 2010.

[6] K. E. Laver, S. George, S. Thomas, J. E. Deutsch and M. Crotty, "Virtual reality for stroke rehabilitation", Cochrane Database Syst Rev, vol. 9, n. 2, pp. 101-107, 2011.

[7] M. K. Holden, "Virtual environments for motor rehabilitation: review", Cyberpsychol \& Behav, vol. 8, n. 3, pp. 187-211, 2005.

[8] H. Regenbrecht, G. McGregor, C. Ott, S. Hoermann, T. Schubert, L. Hale, L. Hoermann, B. Dixon and E. Franz, "Out of reach? a novel AR interface approach for motor rehabilitation", Proceedings of the 10th IEEE international symposium on mixed and augmented reality (ISMAR 2011), pp. 219-228, Oct 2011.

[9] G. Burdea, T. Blois, E. Ching, J. Halton, B. Lopetinsky and V. Drysdale, "Tutorial 1:Nintendo Wii-based rehabilitation", Proceedings of Virtual Rehabilitation, Vancouver, Canada, 2008.
[10] M. Kuttuva, R. Boian, A. Merians, G. Burdea, M. Bouzit, J. Lewis and D. Fensterheim, "The Rutgers Arm: An upper-extremity rehabilitation system in virtual reality", CyberTherapy Behavior, vol. 9, n. 2, pp. 148$152,2006$.

[11] S. J. Housman, K. M. Scott and D. J. Reinkensmeyer, "A randomized controlled trial of gravity-supported, computer-enhanced arm exercise for individuals with severe hemiparesis", Neurorehabilitation Neural Repair, vol. 23, n.5, pp. 505-514, 2009.

[12] A. Gaggioli, F. Morganti, R. Walker, A. Meneghini, M. Alcaniz, J. A. Lozano, J. Montesa, J. A. Gil and G. Riva, "Training with ComputerSupported Motor Imagery in Post-Stroke Rehabilitation", CyberPsychology \& Behavior, vol. 7, n. 3, pp. 327-332, 2004.

[13] J. A. Lozano, J. Montesa, M. C. Juan, M. Alcaiz, B. Rey, J. A. Gil, J. M. Martinez, A. Gaggioli and F. Morganti, "VR-Mirror: A Virtual Reality System for Mental Practice in Post-Stroke Rehabilitation", Lecture Notes in Computer Science, pp. 241-251, 2005.

[14] G. A. Assis, A. G. Correa, M. B. R. Martins, W. G. Pedrozo and R. D. Lopes, "An augmented reality system for upper-limb post-stroke motor rehabilitation: a feasibility study", Disability and Rehabilitation: Assistive Technology, vol. 4, pp. 1-8, 2014.

[15] J. Decety, "The neurophysiological basis of motor imagery", Behavioural Brain Research, vol. 77, pp. 45-52, 1996.

[16] A. Klein and G. A. Assis, "A markeless augmented reality tracking for enhancing the user interaction during virtual rehabilitation", XV Symposium on Virtual and Augmented Reality (SVR), pp. 117-124, 2013 May, Cuiaba, Brazil, 2013.

[17] G. Kristo, G. Rutten, M. Raemaekers, B. Gelder, S. Rombouts and N. F. Ramsey, "Task and Task-Free FMRI Reproducibility Comparison for Motor Network Identification”, Human Brain Mapping, vol. 35, pp. 340352, 2014.

[18] D. Attwell and C. Iadecola, "The neural basis of functional brain imaging signals", Trends in Neurosciences, vol. 25, n. 12, pp. 621-625, 2002.

[19] D. M. Cole, S. M. Smith and C. F. Beckmann, "Advances and pitfalls in the analysis and interpretation of resting-state FMRI data", Front Syst Neurosci. vol. 4, n. 8, 2010.

[20] C. F. Beckmann, M. DeLuca, J. T. Devlin and S. M. Smith, "Investigations into resting-state connectivity using independent component analysis", Philosophical Transactions of the Royal Society B-Biological Sciences, vol. 360, n. 1457, pp. 1001-1013, 2005.

[21] B. Biswal, F. Z. Yetkin, V. M. Haughton and J. S. Hyde, "Functional connectivity in the motor cortex of resting human brain using echoplanar MRI", Magn Reson Med, vol. 34, pp. 537-541, 1995.

[22] S. Y. Chiou, R. Y. Wang, K. K. Liao, Y. T. Wu, C. F. Lu and Y. R. Yang, "Coactivation of primary motor cortex ipsilateral to muscles contracting in a unilateral motor task", Clin Neurophysiol, vol. 124, pp.13531363, 2013.

[23] G. A. Assis, A. G. D. Correa, C. J. N. Vaz and R. D. Lopes, "An Augmented Reality System For Post-Stroke Rehabilitation Using Mental Practice", IADIS International Conference on Computer Graphics and Visualization, July, PP. 24-26, Amsterdam, Holland, 2008.

[24] B. M. Campos, A. C. Coan, C. L. Yasuda, R. F. Casseb and F. Cendes, "LargeScale Brain Networks Are Distinctly Affected in Right and Left Mesial Temporal Lobe Epilepsy", Human Brain Mapping, vol. 37(9), pp. 31373152, 2016.

[25] N. Tzourio-mazoyer, B. Landeau, D. Papathanassiou, F. Crivello, O. Etard, N. Delcroix, B. Mazoyer and M. Joliot, "Automated Anatomical Labeling of Activations in SPM Using a Macroscopic Anatomical Parcellation of the MNI MRI Single-Subject Brain”, NeuroImag, vol. 15, pp. 273289, 2002.

[26] J. O. Shin, H. Ryu, S. and H. Jang, "Task-specific interactive game-based virtual reality rehabilitation system for patients with stroke: a usability test and two clinical experiments", Journal of Neuro Engineering and Rehabilitation, vol. 11, n. 32, 2014.

[27] Brandao, A. F. ; Casseb, R. F. ; Almeida, S. R. M. ; Assis, G. A. ; Camargo, A. F. B. ; Li, L.M. ; Castellano, G. . Investigation of fMRI protocol for evaluation of Gestural Interaction applied to upperlimb motor improvement. SBC JOURNAL ON 3D INTERACTIVE SYSTEMS , 2019.

[28] M. Zanon, G. Novembre, N. Zangrando, L. Chittaro and G. Silani, "Brain activity and prosocial behavior in a simulated life-threatening situation", NeuroImage, Apr., 2014.

[29] Kim, S.M. (2014). Virtual reality therapy for Internet gaming disorder. Alcohol and Alcoholism, Oxford, 49. 
[30] Fajnerov, I., Gregu, D., Hlinka, J., Nekovov, T., koch, A., Ztka, T., Horek, J. (2018). Could Prolonged Usage of GPS Navigation Implemented in Augmented Reality Smart Glasses Affect Hippocampal Functional Connectivity? BioMed Research International, 2018, 110. https://doi.org/10.1155/2018/2716134.

[31] Wong CW, Olafsson V, Plank M, Snider J, Halgren E, Poizner H, et al. (2014) Resting-State fMRI Activity Predicts Unsupervised Learning and Memory in an Immersive Virtual Reality Environment. PLoS ONE 9(10): e109622. https://doi.org/10.1371/journal.pone.0109622.

[32] Boydet al. (2015). Mitii ABI: Study protocol of a randomised controlled trial of a web-based multi-modal training program for children and adolescents with an Acquired Brain Injury (ABI). BMC neurology. DOI 10.1186/s12883-015-0381-6.

[33] A. R. Carter, G. L. Shulman and M. Corbetta, "Why use a connectivitybased approach to study stroke and recovery of function?", NeuroImage, vol. 62, pp. 2271-2280, 2012.

[34] C. Grefkes and G. R. Fink, "Connectivity-based approaches in stroke and recovery of function", Lancet Neurol., vol. 13, pp.206-216, 2014.

[35] G. A. James, Z. L. Lu, J. W. VanMeter, K. Sathian, X. P. P. Hu and A. J. Butler, Changes in resting state effective connectivity in the motor network following rehabilitation of upper extremity post stroke paresis. Top Stroke Rehabil, vol. 16, pp. 270-281, 2009.

[36] C. Grefkes, D. A. Nowak, S. B. Eickhoff, M. Dafotakis, J. Kust, H. Karbe H and G.R. Fink, "Cortical connectivity after subcortical stroke assessed with functional magnetic resonance imaging", Ann. Neurol, vol. 63, pp. 236-246, 2008.

[37] N. U. Dosenbach, D. A. Fair, F. M. Miezin, A. L. Cohen, K. K. Wenger, R. A. Dosenbach, M. D. Fox, A. Z. Snyder, J. L, Vincent, M. E. Raichle, B. L. Schlaggar and S. E. Petersen, "Distinct brain networks for adaptive and stable task control in humans", Proc. Natl. Acad. Sci. USA, vol. 104, n. 26, pp. 1107311078, 2007.

[38] A. R. Carter, K. R. Patel, S. V. Astafiev, A. Z. Snyder, J. Rengachary, M. L. Strube, A. Pope, J. S. Shimony, C. E. Lang, G. L. Shulman and M. Corbetta, "Upstream dysfunction of somatomotor functional connectivity after corticospinal damage in stroke", Neurorehabil Neural Repair, vol. 26, pp.7-19, 2012b.

[39] Leon, Andrew C. ; Davis, Lori L. ; Kraemer, Helena C. The role and interpretation of pilot studies in clinical research. Journal of Psychiatric Research, 2011, Vol.45(5), pp.626-629. 